\title{
Direct Composite Veneering for Anterior Rehabilitation Using Customized Single Tooth Template System- C Veneers- A Unique Approach
}

\author{
Manjunathmandhiradoss Ramaiah ${ }^{1}$, Sudhapriya Arumugaraj ${ }^{2}$, Jenisteffy Shelton ${ }^{3}$
}

\author{
${ }^{1}$ Department of Conservative Dentistry and Endodontics, TKMCH, Thoothukudi, Affiliated to the Dr. MGR Medical \\ University, Tamilnadu, India. ${ }^{2}$ Department of Conservative Dentistry and Endodontics, TKMCH, Thoothukudi, \\ Affiliated to the Dr. MGR Medical University, Tamilnadu, India. ${ }^{3}$ Department of Conservative Dentistry and \\ Endodontics, TKMCH, Thoothukudi, Affiliated to the Dr. MGR Medical University, Tamilnadu, India.
}

\section{INTRODUCTION}

Aesthetic dentistry is an important field in dental practice which provides fantastic solutions to enhance patient's appearance. The common problems that have a big impact on patient's smile are "spacing" and "discoloration" thus the patient seeks aesthetic dental treatment ${ }^{1}$. Anterior teeth rehabilitation provides excellent success results when patient and doctor have a good rapport and when proper technique with correct handling of material is executed. Veneering is the most commonly used method for anterior rehabilitation since 1980's. ${ }^{2}$ Veneers can be fabricated as indirect restoration using porcelain or composite resin or as direct restoration using composite resin. ${ }^{3}$ Direct veneering have certain advantages over indirect veneering 4 . However, the final result of direct composite veneering depends entirely on handling characteristics and artistic skill of dentist. The clinical difficulty of sculpting, length of time to perform has limited its use, of choice for indirect restoration. To overcome this, direct veneering performed with template assisted veneers has opened this treatment modality to any dentist regardless of skill level or artistic ability with less chairside time. ${ }^{5}$

Template assisted veneers can be done either as tray templates or single tooth template system. Single tooth template system concentrates on individual tooth and provides good aesthetic result compared to tray templates. The use of comprehensive (stock) single tooth template system available in market does not provide exact adaptability to individual tooth, interdental accuracy, perfect shape and symmetry and thus finishing consumes more time. So, in order to overcome these disadvantages, an alternative approach with $\mathrm{C}$-veneers -customized single tooth template system has been introduced in these cases which delivers fabulous aesthetic results. ${ }^{5}$ Rehabilitation of anterior teeth with veneering plays an important role in aesthetic or cosmetic dentistry to create a beautiful smile design. Direct composite veneering provides symmetrical and harmonious restoration of the teeth and preserves sound tooth structure when compared to indirect veneering. However, the use of direct composite veneering is very technique sensitive, needs operator skill and more chairside time to sculpt the shape and symmetry of teeth to get efficient results. This article presents 2 case reports of aesthetic management of anterior teeth spacing and discoloration with direct composite veneering in an innovative approach using customized single tooth template system. This method improves patient's aesthetic needs by providing perfect shape and anatomy of individual tooth with accurate interdental contact and contours, consumes less material and chairside time making it more economical, thereby enhancing patient satisfaction.
Corresponding Author:

Dr. Manjunathmandhiradoss Ramaiah, Senior Assistant Professor,

Department of Dental surgery,

Thoothukudi govt. Medical College \& Hospital, Thoothukudi 628003.

Affliated to the Tamilnadu Dr. M.G.R.Medical University

Tamilnadu, India.

E-mail:mr.manjunathmds@gmail.com

DOI: $10.14260 / j e m d s / 2020 / 104$

Financial or Other Competing Interests: None.

How to Cite This Article:

Ramaiah M, Arumugaraj S, Shelton J. Direct composite veneering for anterior rehabilitation using customized single tooth template system- cveneers- a unique approach. J. Evolution Med. Dent. Sci. 2020;9(07):460-464, DOI:
Submission 05-12-2019,

Peer Review 22-01-2020,

Acceptance 29-01-2020,

Published 17-02-2020. 


\section{Case 1}

A 30 years old female patient reported to our hospital with a complaint of spacing in her upper front teeth. On examination, generalised spacing was noted in maxillary anterior teeth with class I malocclusion. Thus, the diagnosis was made as maxillary anterior spacing [fig-1].Initial procedure starts with impression of the teeth which was made using alginate impression material (Tropicalgin, Zhermack, Italy). A primary cast model made of dental stone type 3 (Ultra-Stone, Kalabhai Karson Private Limited, Mumbai) was made [fig-2]. A composite veneer replica, mock wax up fabrication was done covering the labial surface of maxillary anteriors excluding canine and covering the interdental spaces [fig-2]. The mock wax up replica was used to generate templates that would approximate the tooth contours. For accurate reproduction of the patient's teeth, a polyvinyl impression ( putty) (Aquasil, Dentsply, India) was taken from the primary cast with mock wax up, which was then converted as a secondary cast model with die stone.
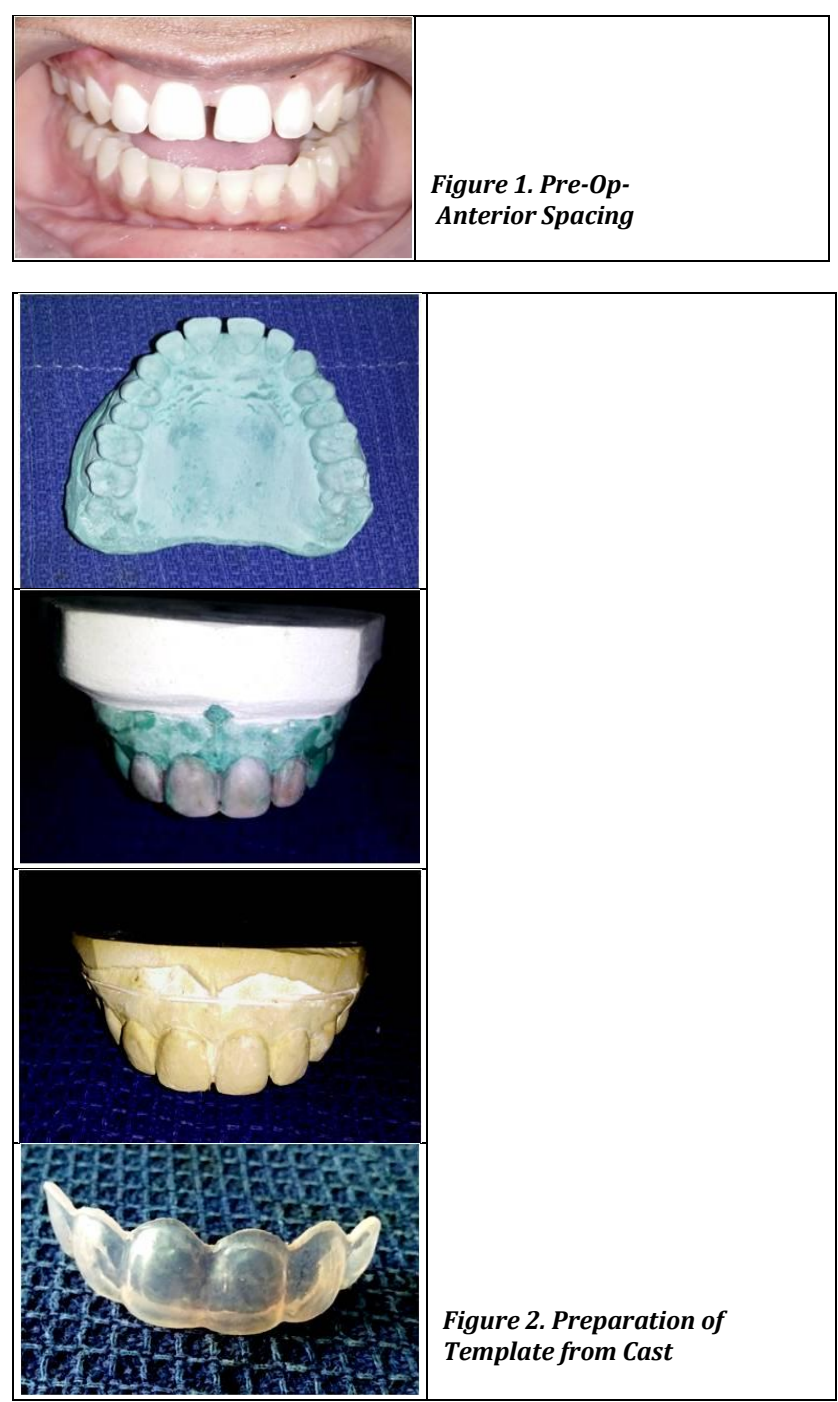

(Ultrarock, Kalabhai Karson Private Limited, Mumbai) [fig2]. A template for maxillary anteriors from canine to canine was fabricated in the laboratory with flexible, transparent, thermoplasticised material which becomes stiff when processed in vacuum pressure (Avac R clear thermoforming sheets, Jaypee general agencies, India) from the secondary cast model [fig-2].

This template was then individually separated carefully in the interproximal region for each tooth as single tooth templates without altering its contours using diamond disc. Individual templates were then attached with plastic holders, which were cut from small clear transparent disposable plastic spoons. The holders were attached at the marked midline in the incisal end of individual template positioning it centric so that there will not be any difficulty in orientation, thus customized single tooth templates were made for each tooth. This type of customized single tooth templates, C-veneers [fig$3]$ create veneers with predictable shape and symmetry and with proper contact and contour with less clinical chair time.

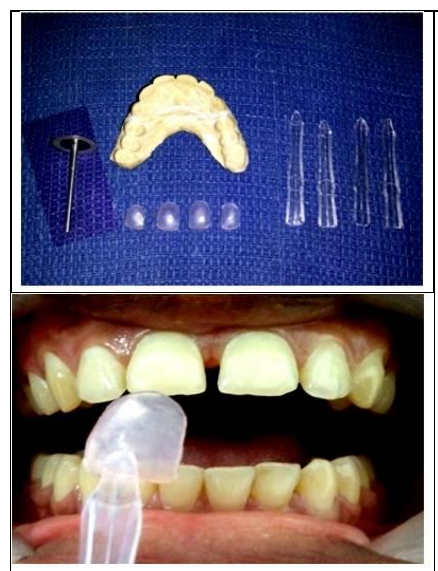

Figure 3. Preparation of Single Tooth Template $C$-Veneer

Patient was made ready. Sectioned matrix using mylar strips and wedges were used to separate the tooth interproximally. An aesthetic nano-filled flowable composite material (TeEconom Flow, Ivoclar Vivadent, Schaan, Liechtenstein) was chosen as it exhibits natural tooth opalescence and fluorescence. Shade selection was done using composite shade guide and the shade selected was "A2". Then the upper anterior teeth excluding canine were acid etched with $37 \%$ phosphoric acid for 20 seconds [fig-4] and then thoroughly rinsed for 10 seconds with water and air dried. Teeth were isolated and bonding agent was applied. After thinning of the bonding agent for 5 seconds it was light cured for 10 seconds.

The template was made ready. Flowable light cure composite with selected shade of A2 was applied over the template and to the entire restorable surface each tooth [fig 4]. The composite filled template was seated over each tooth separately and gently pressed [fig-4]. Each tooth filled with composite was cured over the template for 20 seconds. The template was removed from each tooth after curing without disturbing the composite veneered teeth. The untrimmed direct composite veneers were noted with excess flow material in the gingival margins and teeth shapes were perfectly formed with proper interproximal contact [fig-5]. The proper adaptation of mylar strips and wedges interproximally gives a proper contour and doesn't allow excess flow in the interproximal region. Excess composite flash materials were removed without stress. Final veneers of the teeth were trimmed and polished [fig-5]. 


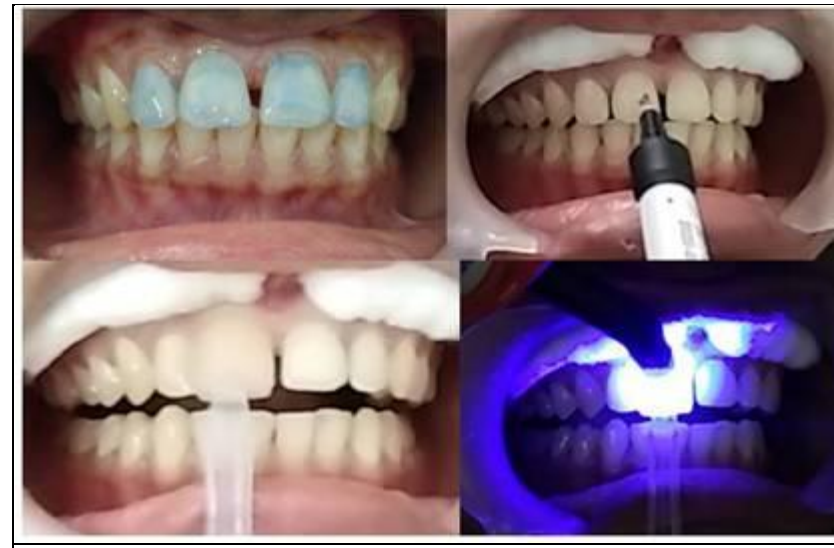

Figure 4. Teeth were Etched, Rinsed, Bonded. Matrix and Wedges were Placed Followed by Composite Application with Template

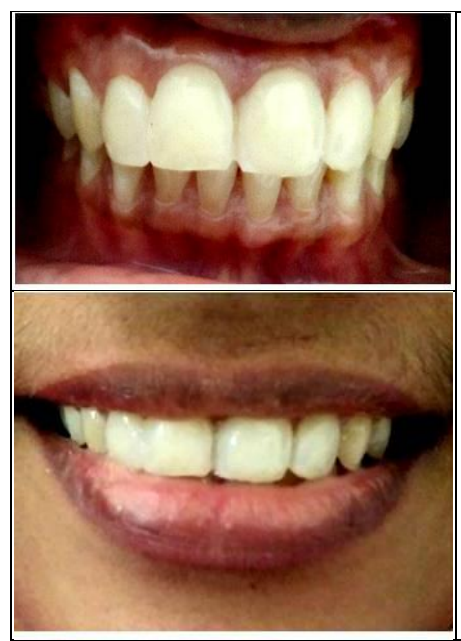

Figure 5. Post-Op Aesthetic Smile After Finishing and Polishing

\section{Case 2}

A 17-year-old female patient reported to our hospital with a complaint of yellowish discoloration of all teeth since childhood. Medical history and family history were unremarkable. On intraoral examination, patient had permanent dentition with no missing teeth, upper and lower teeth showed yellowish brown discoloration with worn off cusps in molars [fig-6].

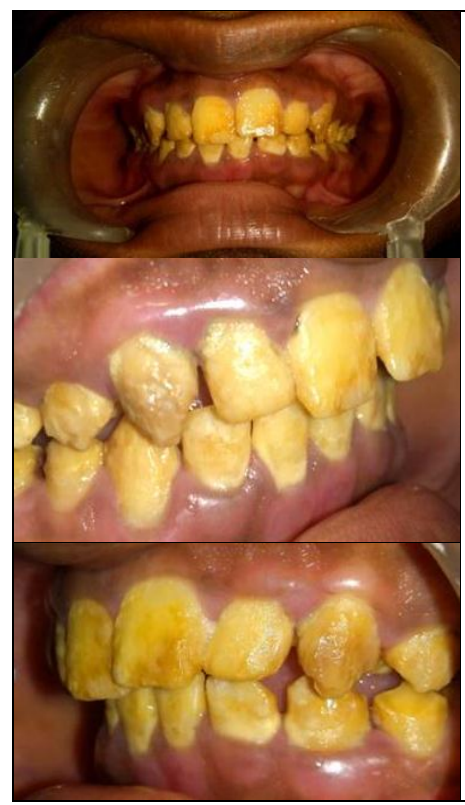

Figure 6. Pre-Op- $A$ Case of Amelogenesis Imperfecta
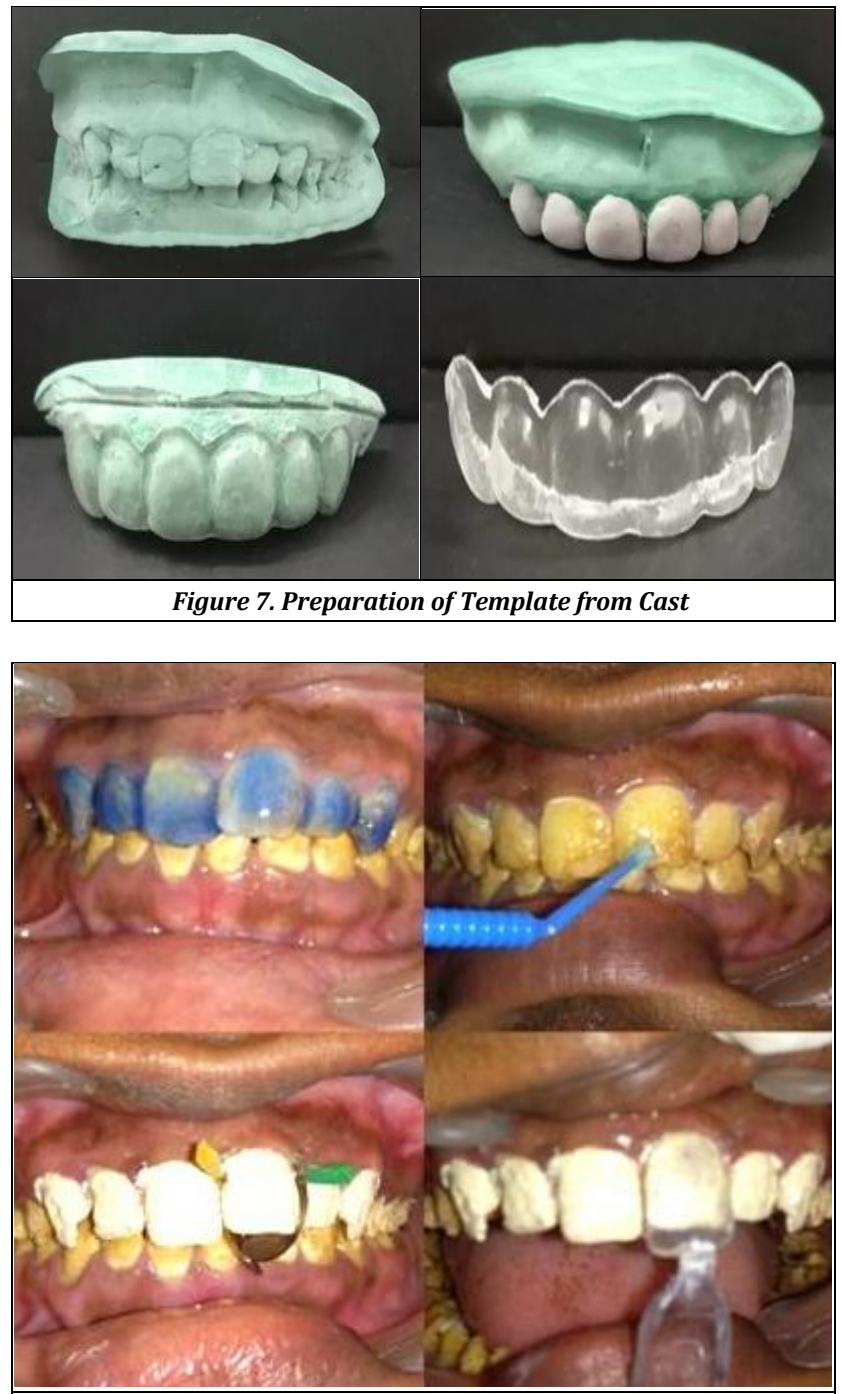

Figure 8. Teeth were Etched, Rinsed, Bonded. Matrix and Wedges were Placed Followed by Application of Opaquer and Template was Prepared

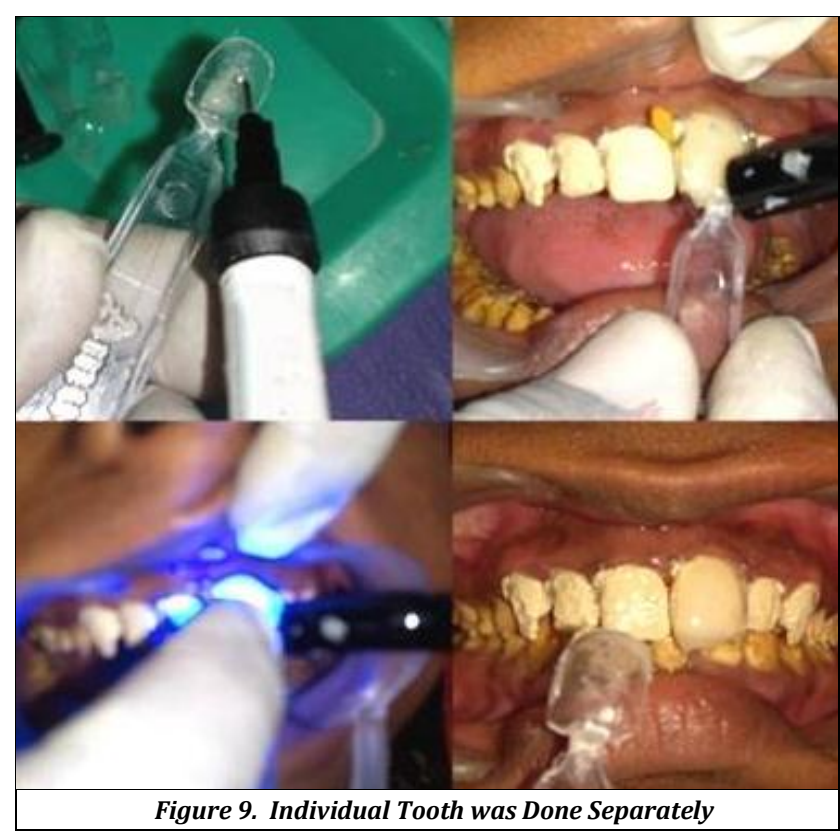

On the basis of clinical examination, provisional diagnosis of hypoplastic AI was made and an orthopantomogram (OPG) was advised which showed presence of a thin layer of enamel. ${ }^{6}$ 
Final diagnosis of hypoplastic AI was made and was planned for anterior rehabilitation with $\mathrm{C}$-veneers. The anterior rehabilitation procedure was done similar to case report-1. Mock wax up fabrication was done on the primary cast, which was then converted to a secondary cast model followed by fabrication of thermoplasticised template which was then sectioned and attached with holders. [fig-7].

The templates which were loaded with A2 flowable composite were applied to individual tooth after acid etching, rinsing, bonding and application of opaquer, the restoration was done [fig-8,9].

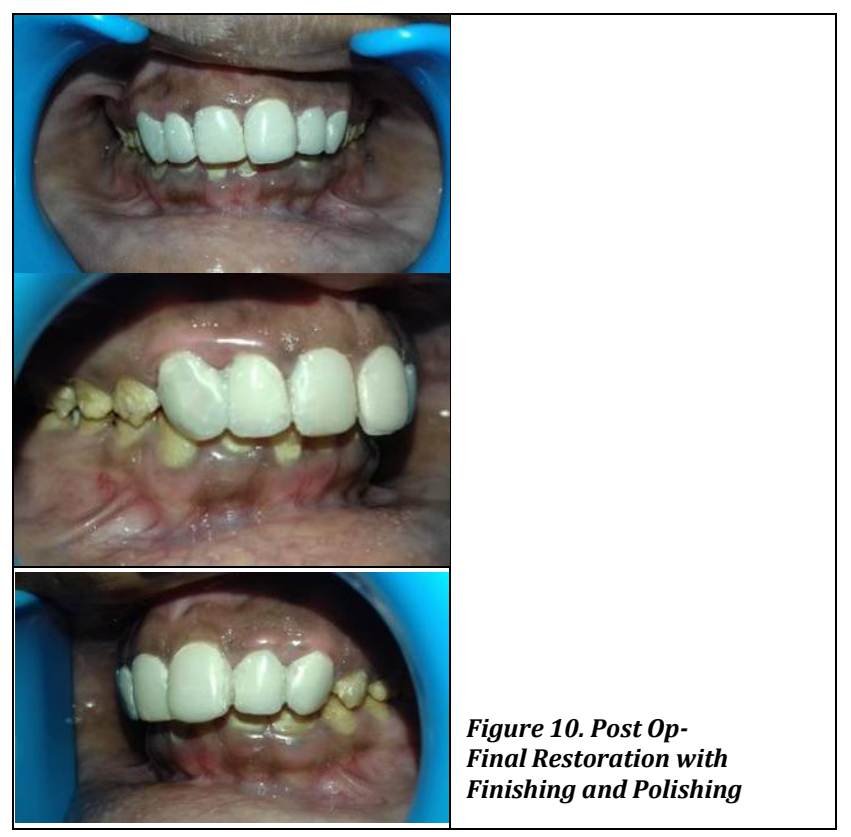

Followed by finishing and polishing of the restoration by trimming of excess composite [fig- 10].

\section{DISCUSSION}

Smile raises the face value and smile design is one of the main areas of cosmetic dentistry [7]. Smile designing is the process of improving the appearance of a compromised smile through cosmetic dental procedure. The need for aesthetics among the people motivates them to seek dental treatment which is often dictated by cultural, ethnic and individual preferences. The aim of treatment in this case is to restore patient's aesthetics and self-esteem. Historically, anaesthetic anterior teeth were improved using full crowns but as dentistry has progressed, veneers have become a more conservative approach. ${ }^{[8]}$

In case report- 1 the patient had anterior spacing and in case report-2 the patient had discoloration of all teeth in order to establish both functional and aesthetic integrity, veneers were considered. The advantages of direct composite veneers are minimal/non- tooth preparation, single appointment procedure, less fracture rates and easy to repair, they are strong and durable, have similar abrasion rates as natural tooth structures and mainly is "cost effective."[9] Moreover polishing of direct composite veneers is easy, marginal adaptation is good. Whereas indirect veneers require reshaping of natural teeth in order to fit the veneer, tooth surface removal leading to sensitivity, multiple visits, failures due to debonding \& fracture, they need temporary restoration and cost more. ${ }^{[4,8]}$ In these cases, the use of conservative direct composite veneers provided symmetrical and harmonious restoration of the teeth.

With the introduction of veneer template system which allows a placement protocol with greater predictability and ease of application, the use of direct composite resin veneers is expected to increase in the popularity among clinicians and patients.[10] The template system provides perfectly contoured facial surface, lack of oxygen inhibited layer. It consumes less time, easy to handle, requires no additional polishing steps. It is minimally invasive and also affordable [11]. However, when we use template as single unit tray for anteriors, the contact and contours cannot be properly established, excess flow of the material cannot be controlled, difficulty in adapting to individual tooth, more wastage of material and consumes more time for finishing and polishing.

The readymade stock Uveneer System available in the market is a clear template kit that provides preformed facial surfaces of maxillary incisors, canines and premolars. The size of the Uveneer templates is made to match ideal $80 \%$ widthto-length ratios.[12] But the customized templates Cveneer provide perfect width to length ratios and enhance smile design proportion. The thickness of the customized translucent thermoforming sheets is $1.5 \mathrm{~mm}$ which allows sufficient polymerizing light to pass through it.

The advantages of customized individual or single tooth templates provide excellent individual tooth adaptability with high interdental accuracy, establish good shape and symmetry with proper separation by matrix and wedge. It also provides minimal excess, consumes less material and allows operator to concentrate on individual tooth. Thus finishing and polishing are minimal and consumes less chair time.

On material aspect, introduction of nanotechnology to flowable composite permitted the development of a composite with the mechanical properties, wear resistance, strength, enhanced polishability, excellent polish retention, translucency of a conventional resin composite and keeping elasticity, adaptation, and favorable handling characteristics of flowable composite with minimized polymerization shrinkage by almost $20 \%$.[13] Studies used flowable composite in noncarious cervical lesions, and there was no statistical or clinical differences between flowable and conventional composites. therefore, the use of a flowable resin composite in non-carious cervical lesions was recommended as being beneficial.[14]

Hence in this case flowable composites (TeEconom flow, Ivoclarvivadent, Schaan, Liechtenstein) was preferred over packable as they are less viscous, easily injectable, wet the surface more fully than packable composite. They create more intimate adaptation between tooth and restorative material and also have good seal. Flowable composites also form layers of minimal thickness, so eliminates air entrapment. It has high flexibility, so less likely to be displaced in stress concentrated areas. It is radio opaque, with increased translucency and polishability.[9] 


\section{CONCLUSIONS}

Case selection with regard to aesthetic correction of misalignment, form, colour, and spacing issues of anterior teeth, and also carious lesions of anterior teeth, is the most important factor. ${ }^{8}$ When treating patients with complicated aesthetic needs, direct mock up technique facilitates predictable aesthetic results ${ }^{15}$ and the laboratory fabricated templates is of course simple, time saving, low stress and provides perfect dental anatomy. ${ }^{5}$ Hence, C-veneers customized single tooth template system is proven to give fabulous and easy restoration of anterior teeth as an alternative to hand sculpted direct composite veneers and other market available stock template systems. So, enlighten the smile by beautifying your teeth and let the world smile back to you. ${ }^{11}$

\section{REFERENCES}

[1] Sowmya K, Dwijendra KS, Pranitha V, et al. Esthetic rehabilitation with direct composite veneering: a report of 2 cases. Case Rep Dent 2017;2017:7638153.

[2] Re D, Augusti G, Amato M, et al. Esthetic rehabilitation of anterior teeth with laminates composite veneers. Case Report. Dent 2014;2014:849273.

[3] Mangani F, Cerutti A, Putignano A, et al. Clinical approach to anterior adhesive restorations using resin composite veneers. The European Journal of Esthetic Dentistry 2007;2(2):188-209.

[4] Wakiaga J, Brunton P, Silikas N, et al. Direct versus indirect veneer restorations for intrinsic dental stains. Review. Cochrane Database of Systematic Reviews 2004;(1):CD004347.
[5] Goldstein MB. Template-assisted direct composite veneers. Dent Today 2010;29(2):124, 128-9.

[6] Chaudhary M, Dixit S, Singh A, et al. Amelogenesis imperfect: report of a case and review of literature. J Oral Maxillofac Pathol 2009;13(2):70-7.

[7] Nalbandian S, Millar BJ. The effect of veneers on cosmetic improvement. British Dental Journal 2009;207(2):72-3.

[8] Andykayayan S. Bonded composites versus ceramic veneers. BDA Evidence Summary 2014:p 1-7.

[9] Lal SM. Jagadish S. Direct composite veneering technique producing a smile design with a customized matrix. Journal of Conservative Dentistry 2006;9(3):87-92.

[10] Morgan J. Predictable direct composite resin veneers: a template system makes it easier. Dental Materials 2018.

[11] Thomas D, Hari K, Mathew J, et al. Esthetic correction with laminate veneers - a case series. Journal of Dental Sciences 2017.

[12] Lowe RA. Simplifying direct composite veneer placement. Dent Today 2015;34(5):98, 100-3.

[13] Baroudi K, Rodrigues JC. Flowable resin composites: a systematic review and clinical considerations. Journal of Clinical and Diagnostic Research 2015;9(6):ZE18-ZE24.

[14] Shaalan O0, Abou-Auf E, El Zoghby AF. Clinical evaluation of flowable resin composite versus conventional resin composite in carious and noncarious lesions: systemic review and meta-analysis. Journal of Conservative Dentistry 2017;20(6):380-5.

[15] Reshad M, Cascione D, Magne P. Diagnostic mock-ups as an objective tool for predictable outcomes with porcelain laminate veneers in esthetically demanding patients: a clinical report. J Prosthet Dent 2008;99(5):333-9. 